Galban CJ, Boes JL, Bule M, et al. Parametric response mapping as an indicator of bronchiolitis obliterans syndrome after hematopoietic stem cell transplantation. Biol Blood Marrow Transplant 2014; 20: 1592-1598.

4 Buist AS, McBurnie MA, Vollmer WM, et al. International variation in the prevalence of COPD (the BOLD Study): a population-based prevalence study. Lancet 2007; 370: 741-750.

5 Miller MR, Hankinson J, Brusasco V, et al. Standardisation of spirometry. Eur Respir J 2005; 26: 319-338.

6 Crapo RO, Casaburi R, Coates AL, et al. Guidelines for methacholine and exercise challenge testing - 1999. This official statement of the American Thoracic Society was adopted by the ATS Board of Directors, July 1999. Am J Respir Crit Care Med 2000; 161: 309-329.

7 Dyer C. The interaction of ageing and lung disease. Chron Respir Dis 2012; 9: 63-67.

8 Janssens JP, Pache JC, Nicod LP. Physiological changes in respiratory function associated with ageing. Eur Respir J 1999; 13: 197-205.

9 Lee KW, Chung SY, Yang I, et al. Correlation of aging and smoking with air-trapping at thin-section CT of the lung in asymptomatic subjects. Radiology 2000; 214: 831-836.

10 Gevenois PA, Scillia P, de Maertelaer V, et al. The effects of age, sex, lung size, and hyperinflation on CT lung densitometry. AJR Am J Roentgenol 1996; 167: 1169-1173.

11 Wang Q, Takashima S, Wang JC, et al. Prevalence of emphysema in individuals who underwent screening CT for lung cancer in Nagano prefecture of Japan. Respiration 2001; 68: 352-356.

12 Camiciottoli G, Cavigli E, Grassi L, et al. Prevalence and correlates of pulmonary emphysema in smokers and former smokers. A densitometric study of participants in the ITALUNG trial. Eur Radiol 2009; 19 : 58-66.

13 Matsuoka S, Kurihara Y, Yagihashi K, et al. Quantitative assessment of air-trapping in chronic obstructive pulmonary disease using inspiratory and expiratory volumetric MDCT. AJR Am J Roentgenol 2008; 190: 762-769.

14 Bommart S, Marin G, Bourdin A, et al. Relationship between CT air-trapping criteria and lung function in small airway impairment quantification. BMC Pulm Med 2014; 14: 29.

15 Schroeder JD, McKenzie AS, Zach JA, et al. Relationships between airflow obstruction and quantitative CT measurements of emphysema, air-trapping, and airways in subjects with and without chronic obstructive pulmonary disease. AJR Am J Roentgenol 2013; 201: W460-W470.

\title{
Accuracy of chest high-resolution computed tomography in diagnosing diffuse cystic lung diseases
}

To the Editor:

The diffuse cystic lung diseases (DCLDs) are a group of pathophysiologically heterogeneous processes characterised by the presence of multiple, thin-walled, air-filled spaces within the pulmonary parenchyma [1]. The differential diagnosis of DCLDs includes lymphangioleiomyomatosis (LAM), follicular bronchiolitis (FB), lymphocytic interstitial pneumonia (LIP), Birt-Hogg-Dubé syndrome (BHD), pulmonary Langerhans cell histiocytosis (PLCH), amyloidosis, light chain deposition disease, cystic metastases, infectious entities such as Pneumocystis, and other aetiologies [2]. Bronchiectasis and bullous changes seen in chronic obstructive pulmonary disease can also produce high-resolution computed tomography (HRCT) patterns that mimic the DCLDs.

The utility of HRCT in the diagnosis of LAM and differentiation from other DCLDs is not completely defined. According to the European Respiratory Society (ERS) guidelines, characteristic HRCT features along with a compatible clinical history are sufficient to confidently diagnose LAM, without the need for a tissue biopsy [3]. However, previously reported accuracy rates for diagnosing LAM based on HRCT findings may not be sufficient in an era when interventions with substantial risks are becoming available. Two prior studies have reported accuracy rates of $72-84 \%$ in diagnosing LAM based on imaging characteristics alone $[4,5]$. The aim of our study was to determine the diagnostic accuracy of HRCT evaluation by radiologists and pulmonologists, at various levels of expertise, in patients with DCLDs presenting to referral centres.

We retrospectively obtained HRCTs from 89 patients referred to LAM Foundation Clinics at the University of Cincinnati (Cincinnati, OH, USA), Mayo Clinic Rochester (Rochester, MN, USA) and National Kinki-Chou Hospital (Osaka, Japan) for further evaluation of DCLDs. All scans were non-contrast HRCTs and only thin section (1-3 mm) images were employed in the analysis. Patient 
identifiers were removed and the digital image files and a DICOM viewer (Santesoft, Athens, Greece), with full scrolling and magnification capabilities, were distributed to all reviewers. When necessary, abdominal cuts of the HRCT were removed to ensure that pathognomonic abdominal features, such as the presence of angiomyolipomas, would not influence the interpretation. The scans were analysed by three expert thoracic radiologists, and 12 pulmonary physicians with varying levels of expertise, subclassified as DCLD expert pulmonologists $(n=5)$, general pulmonologists $(n=4)$ and pulmonary fellows $(n=3)$.

Observers were asked to record the most likely diagnosis and degree of confidence (confident or not confident). Observers were blind to all clinical or pathological data. Images used for analysis were exclusively derived from patients with definite diagnoses established by biopsy, genetic testing or professional society guidelines. The results were used to calculate sensitivity and specificity of HRCT based diagnoses. In addition, inter-observer agreement among the various groups was calculated using the Fleiss kappa determination. All analyses were conducted using Microsoft Excel and SAS for Windows version 9.3 (Cary, NC, USA).

LAM was the most common disease in our study (45 out of 89 cases). Other cases included: PLCH $(n=18)$, BHD $(n=5)$, LIP/FB $(n=5)$, normal $(n=5)$, emphysema $(n=3)$, amyloidosis $(n=3)$, pleuropulmonary blastoma $(n=1)$, non-specific interstitial pneumonia $(n=1)$, hypersensitivity pneumonitis $(n=2)$, and lymphangiomatosis $(\mathrm{n}=1)$.

Expert radiologists correctly diagnosed LAM in 41 (91\%) out of 45 cases, and when confident, in 34 (98\%) out of 35 cases. DCLD expert pulmonologists correctly diagnosed LAM in 39 (86\%) out of 45 cases, and in 36 (95\%) out of 38 cases, when confident. General pulmonologists and pulmonary fellows correctly identified LAM in $79 \%$ and $83 \%$ of cases, respectively (figure 1a).

The accuracy of diagnosing non-LAM DCLDs based on HRCT was lower than LAM. Expert radiologists performed better than pulmonologists in all categories (figure 1b). Expert radiologists diagnosed PLCH with an accuracy of $74 \%$. Pulmonary physicians correctly identified PLCH in $31-58 \%$ of the cases. Interestingly, when confident, the accuracy of PLCH diagnosis by all observers was nearly perfect for the attending physicians (95-100\%), but not for the trainees (69\%).

Expert radiologists were able to distinguish BHD from other DCLDs with an accuracy of 93\%, rising to $100 \%$ when confident. In contrast, pulmonary physicians and trainees were able to correctly diagnose BHD in $35-47 \%$ of the cases. The accuracy of expert radiologists in the diagnosis of LIP/FB was $54 \%$, and $78 \%$ when confident. Pulmonologists were able to correctly diagnose LIP/FB in $10-44 \%$ of the cases.

Overall, expert radiologists performed better than pulmonologists, correctly assigning the DCLD diagnosis in $71(80 \%)$ out of 89 cases $(\mathrm{p}<0.0001)$ and in $54(89.5 \%)$ out of 60 cases when confident. Inter-observer agreement $(\kappa)$ for correct DCLD diagnosis was highest among the expert radiologists $(\kappa=0.82$, almost perfect agreement), followed by expert pulmonologists $(\kappa=0.64$, substantial agreement), pulmonary fellows $(\kappa=0.56$, moderate agreement), and general pulmonologists $(\kappa=0.53$, moderate agreement).

The results of our analysis show that expert radiologists can accurately diagnose DCLDs in a high proportion of cases $(80 \%)$ based on HRCT features alone. The diagnostic accuracy of pulmonologists

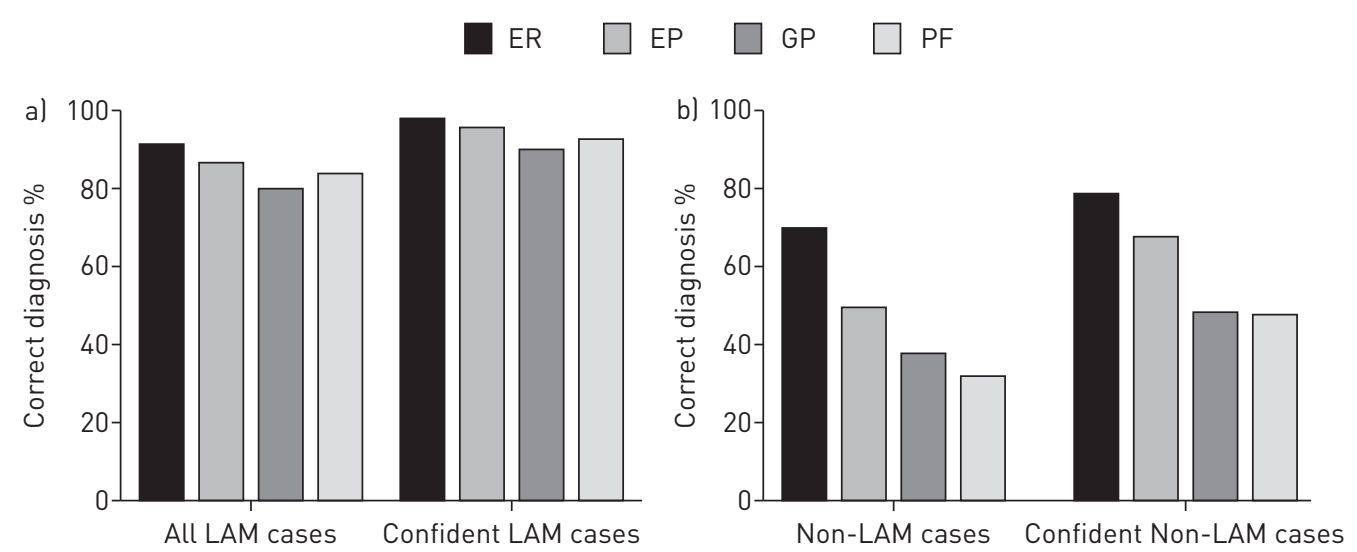

FIGURE 1 Accuracy rates of diagnosing diffuse cystic lung diseases based on high-resolution computed tomography (HRCT) characteristics. a) Accuracy rates of diagnosing lymphangioleiomyomatosis (LAM) based on HRCT images. b) Accuracy rates of diagnosing non-LAM cystic lung diseases based on HRCT images. ER: expert radiologists; EP: expert pulmonologists; GP: general pulmonologists; PF: pulmonary fellows. 
lagged behind radiologists. The accuracy rate for all reviewers in diagnosing LAM based on HRCT features was higher than other DCLDs. Expert radiologists were able to correctly diagnose LAM in $>90 \%$ of cases.

LAM is a female-predominant lung disease caused by mutations in tuberous sclerosis genes and the infiltration of pulmonary parenchyma with abnormal smooth muscle cells [6]. LAM is characterised by the presence of multiple, round, thin-walled diffusely distributed pulmonary cysts, often with normal appearing intervening lung parenchyma [3]. The rationale for pursuing a definite diagnosis has been strengthened of late. A recent randomised controlled trial demonstrated that sirolimus stabilises lung function decline and improves quality of life in patients with LAM [7]. However, effective therapy with sirolimus requires continuous drug exposure and is associated with potential adverse effects. Given the specter of long-term therapy, we favour pursuing the diagnosis until certainty is attained. Therefore, in patients with LAM who are being considered for sirolimus therapy, we recommend a critical appraisal of HRCT findings by an expert, and a diagnosis based on criteria outlined in the ERS LAM Guidelines [6]. In addition, serum vascular endothelial growth factor-D is a useful diagnostic biomarker for LAM that can obviate the need for biopsy in some cases $[8,9]$.

With regard to other DCLDs, expert radiologists correctly diagnosed PLCH in $74 \%$ of the cases, with the accuracy rate increasing to $100 \%$ in confident cases. Based on these results, we submit that HRCT features alone may be sufficient to diagnose PLCH in only the most "typical" cases. Clinicians should consider tissue confirmation for PLCH in atypical cases and in patients considering treatment with agents that have significant side-effect profiles. Although the total number of BHD cases in our study was small, our results suggest that an expert radiologist can diagnose BHD with a high degree of certainty based on HRCT features. However, genetic or skin tissue confirmation is recommended even if the HRCT is characteristic since the diagnosis will commit the patient to lifelong monitoring for renal neoplasms [10].

Our study has several unique features and limitations. Since we restricted the analysis to LAM and other LAM mimics, our dataset was representative of a cohort that would be seen at a DCLD referral centre. This was a retrospective analysis and the number of patients with some of the non-LAM DCLDs, such as BHD and LIP/FB, was small. Another limitation was the lack of availability of standard training and education regarding the diagnostic criteria of various DCLDs for the reviewers. The blinding of evaluators puts them at a disadvantage in making a definitive diagnosis as many DCLDs have characteristic clinical features such as angiomyolipomas in LAM, skin findings of fibrofolliculomas in BHD, and the presence of sicca symptoms and autoantibodies in patients with LIP/FB. Thus the accuracy rate for diagnosing DCLDs is almost certainly higher in real clinical situations than reported here.

We recommend critical review of HRCT features by an expert radiologist when evaluating patients with DCLD, especially when the diagnosis is uncertain or long-term treatment and monitoring programmes are being considered. Tissue or genetic confirmation is recommended if HRCT features are at all atypical or if interventions associated with risk are contemplated.

0 @ERSpublications

Correct diagnosis of diffuse cystic lung diseases is established in most cases by critical review of HRCT features http://ow.ly/NvrCc

Nishant Gupta ${ }^{1}$, Riffat Meraj ${ }^{1}$, Daniel Tanase ${ }^{1}$, Laura E. James ${ }^{2}$, Kuniaki Seyama ${ }^{3}$, David A. Lynch $^{4}$, Masanori Akira ${ }^{5}$, Cristopher A. Meyer, Stephen J. Ruoss ${ }^{6}$, Charles D. Burger, Lisa R. Young, Khalid F. Almoosa ${ }^{8}$, Srihari Veeraraghavan ${ }^{11}$, Alan F. Barker ${ }^{12}$, Augustine S. Lee ${ }^{8}$, Daniel F. Dilling ${ }^{13}$, Yoshikazu Inoue ${ }^{14}$, Corey J. Cudzilo ${ }^{1}$, Muhammad A. Zafar ${ }^{1}$ and Francis X. McCormack ${ }^{1}$

${ }^{1}$ Division of Pulmonary, Critical Care and Sleep Medicine, University of Cincinnati, Cincinnati, OH, USA. ${ }^{2}$ Research Dept, Shriners Hospital for Children - Cincinnati, Cincinnati, OH, USA. ${ }^{3}$ Dept of Respiratory Medicine, Juntendo University, Tokyo, Japan. ${ }^{4}$ Dept of Radiology, National Jewish Health, Denver, CO, USA. ${ }^{5}$ Dept of Radiology, National Kinki-Chuo Chest Medical Center, Osaka, Japan. ${ }^{6}$ Dept of Radiology, University of Wisconsin, Madison, WI, USA. ${ }^{7}$ Division of Pulmonary and Critical Care Medicine, Stanford University School of Medicine, Stanford, CA, USA. ${ }^{8}$ Division of Pulmonary and Critical Care Medicine, Mayo Clinic, Jacksonville, FL, USA. ${ }^{9}$ Division of Allergy, Pulmonary and Critical Care Medicine, Vanderbilt University School of Medicine, Nashville, TN, USA. ${ }^{10}$ Division of Critical Care Medicine, University of Texas Health Science Center, Houston, TX, USA. ${ }^{11}$ Division of Pulmonary, Allergy and Critical Care, Emory University School of Medicine, Atlanta, GA, USA. ${ }^{12}$ Division of Pulmonary and Critical Care, Oregon Health and Science University, Portland, OR, USA. ${ }^{13}$ Dept of Medicine, Loyola University, Chicago, IL, USA.

${ }^{14}$ Dept of Diffuse Lung Diseases and Respiratory Failure, National Hospital Organization Kinki-Chuo Chest Medical Center, Osaka, Japan.

Correspondence: Nishant Gupta, 231 Albert Sabin Way, ML 0564, MSB Room 6053 Cincinnati, OH 45267, USA.

E-mail: guptans@ucmail.uc.edu

Received: March 022015 | Accepted after revision: May 062015 | First published online: July 092015

Disclosures can be found alongside the online version of this article at erj.ersjournals.com 


\title{
References
}

1 Hansell DM, Bankier AA, MacMahon H, et al. Fleischner Society: glossary of terms for thoracic imaging. Radiology 2008; 246: 697-722.

2 Cordier J-F, Johnson SR. Multiple cystic lung diseases. In: J-F Cordier, ed. Orphan Lung Diseases (ERS Monograph). Sheffield, European Respiratory Society, 2011; pp. 46-83.

3 Johnson SR, Cordier J-F, Lazor R, et al. European Respiratory Society guidelines for the diagnosis and management of lymphangioleiomyomatosis. Eur Respir J 2010; 35: 14-26.

4 Bonelli FS, Hartman TE, Swensen SJ, et al. Accuracy of high-resolution CT in diagnosing lung diseases. AJR Am J Roentgenol 1998; 170: 1507-1512.

5 Koyama M, Johkoh T, Honda O, et al. Chronic cystic lung disease: diagnostic accuracy of high-resolution CT in 92 patients. AJR Am J Roentgenol 2003; 180: 827-835.

6 Henske EP, McCormack FX. Lymphangioleiomyomatosis-a wolf in sheep's clothing. J Clin Invest 2012; 122: 3807-3816.

7 McCormack FX, Inoue Y, Moss J, et al. Efficacy and safety of sirolimus in lymphangioleiomyomatosis. $N$ Engl J Med 2011; 364: 1595-1606.

8 Young LR, Vandyke R, Gulleman PM, et al. Serum vascular endothelial growth factor-D prospectively distinguishes lymphangioleiomyomatosis from other diseases. Chest 2010; 138: 674-681.

9 Young L, Lee HS, Inoue Y, et al. Serum VEGF-D a concentration as a biomarker of lymphangioleiomyomatosis severity and treatment response: a prospective analysis of the Multicenter International Lymphangioleiomyomatosis Efficacy of Sirolimus (MILES) trial. Lancet Respir Med 2013; 1: 445-452.

10 Menko FH, van Steensel MA, Giraud S, et al. Birt-Hogg-Dubé syndrome: diagnosis and management. Lancet Oncol 2009; 10: 1199-1206.

\section{Blood stem cell transplantation to treat cystic lung light chain deposition disease}

\author{
To the Editor:
}

Light chain deposition disease (LCDD) is a rare disease resulting from non-amyloid immunoglobulin (Ig) light chain deposition in tissue. In systemic LCDD, plasma cell dyscrasia is common and renal involvement is almost always present, sometimes with damage to other organ systems (cardiac, hepatic and neurological systems). LCDD can be limited to the lungs, presenting as multiple cystic lung disease, nodules or bronchiectasis. Cystic lung related to LCDD (CL-LCDD) was recently described in patients referred for lung transplantation to treat end-stage multiple cystic lung disease [1]. In the reported cases, blood and bone marrow examinations did not reveal clonal plasma cell proliferation. Here we report the first case of CL-LCDD revealing a B-cell extrapulmonary lymphoproliferative disorder and the results of treatment for the underlying haematological disease with autologous peripheral blood stem cell transplantation during the CL-LCDD. Despite respiratory insufficiency, lung transplantation was actually not considered because of the underlying disease.

A 37-year-old Caucasian female was referred to Toulouse University Hospital (Toulouse, France) with cough, dyspnoea and fever of 5 months duration. The patient had sinusitis and bronchitis in infancy, and a close family member had been treated for lymphoma. She had recently quit smoking (smoking history: 10 pack-years). Physical examination revealed only one small indurated cervical lymphadenopathy. Pulmonary function tests showed an obstructive pattern with profound hypoxaemia and altered diffusing capacity of the lung for carbon monoxide: forced expiratory volume in $1 \mathrm{~s}$ (FEV1) was $1700 \mathrm{~mL}$ (56\% predicted), vital capacity was $2500 \mathrm{~mL}$ ( $73 \%$ predicted), total lung capacity was $103 \%$ predicted, diffusing capacity of the lung for carbon monoxide corrected for haemoglobin was $47 \%$ predicted and the partial pressure of oxygen in arterial blood was $42 \mathrm{mmHg}$. A computed tomography (CT) scan revealed diffuse thin-walled cystic formations associated with segmental atelectasis, emphysematous-like changes, bilateral bronchiectasis, and calcified hilar and subcarinal lymph nodes (fig. 1a). Positron emission tomography (PET)-CT revealed minimal uptake in the subcarinal lymph node. Histology of endobronchial biopsies revealed typical LCDD characterised by an inflammatory infiltrate with extracellular amorphous eosinophilic deposits (fig. 1b), negative Congo-red staining and no birefringence under polarised light. Immunofluorescence assays of frozen tissue revealed intense labelling for anti- $\kappa$ light chain antibody. The diagnosis was CL-LCDD.

An extensive search for extrapulmonary organ involvement was negative. Serum densitometry revealed a small monoclonal $\operatorname{IgM} \kappa$ peak $\left(0.29 \mathrm{~g} \cdot \mathrm{L}^{-1}\right)$, with associated hypogammaglobulinaemia $\left(6.64 \mathrm{~g} \cdot \mathrm{L}^{-1}\right)$. 